\title{
MODEL AND ANALYSIS OF DESKTOP WIND TUNNEL
}

\author{
MD. KHALEEL ${ }^{1}$, S. ALKA ${ }^{2}$, \& MARAMPALLI SHILPA ${ }^{3}$
}

${ }^{1,2}$ Assistant Professor, Department of Aeronautical Engineering, MLR Institute of Technology, Hyderabad, Telangana, India

${ }^{3} P G$ Student, Aeronautical Engineer, Hyderabad, Telangana, India

\begin{abstract}
This paper is on model and analysis of desktop wind burrow. Wind Tunnel (WT) is equipment used to research a communication between rigid body flows in WT that can be performed in term of observing physical stream phenomenon, for example, laminar, turbulent streams, vortex flows. It's also able to measure aerodynamic quantities, but the center of attention of this report will be on the model of WT and doing analysis on it. It's about application of similitude theory on the prototypes. The main step is to scale down the shape and dimension of the prototype, or real model into this model. This similitude theory application will save money and space toward industries. The components of $a$ WT and the main objective of each are given below.

The significance of delivering uniform airflow to the test section is considered with respect to the impact of non-uniform flow on the measured performance of equipment, which is shown to be velocity-dependent. A straightforward approach for constructing a WT with great test section flow is described.

KEYWORDS: WT, Vortex Flows \& Velocity-Dependent
\end{abstract}

Received: Nov 19, 2017; Accepted: Dec 09, 2017; Published: Jan 11, 2018; Paper Id.: IJMPERDFEB201857

\section{INTRODUCTION}

My inspiration for this project is obviously to have a valuable knowledge. The inspiration for this project, in Particular is to provide the college with a WT, which it can use in future Experiments. The WT was initially made to be utilized as a major aspect of a lab in which, students would figure the powers on a ping pong ball in wind stream. My goal is to have the tunnel be available for use for a more extensive scope of purposes. It can be modified once again \& used to show understudies about fluid elements. It likewise, can possibly help in the comprehension of the Aerodynamics involved in future understudy projects.

Additionally, it is my goal to manufacture a WT in as low as possible and can save several lakhs of rupees having been made by a under graduate, also keeps the design moderately basic and it is easily understood. This enables it to be more effortlessly altered, without fear of damaging complex and expensive equipment, which might be hard to supplant or repair.

In the WT, the designer can purposely control the stream conditions which impact forces on the a/c. By making wary estimations of the forces on the prototype, the designer can anticipate the forces on the real a/c. Furthermore, by using remarkable analytic techniques, the designer can comprehend and enhance the execution of the $\mathrm{a} / \mathrm{c}$.

WT are intended for a particular reason \& there is a wide assortment of WT sorts \& prototypes. The prototype to be tried in the WT is set in the test segment of the WT. The speed in the test segment is dictated 
by the plan of the WT. The decision of speed extend influences the plan of the WT, because of compressibility impacts

\section{PROBLEM STATEMENT AND DESCRIPTION OBJECTIVES}

The primary goals are to accomplish prototype are:-

- $\quad$ Sketch WT utilizing different parameters.

- Concentrate on the velocity profile by observing stream lines on airfoil.

- $\quad$ Analyze the aerodynamics coefficient for various mach numbers.

\section{CALCULATIONS}

Testing Chamber: To fix the picked prototype measurements, the test segment measurements are picked as:

$15 \times 15 \mathrm{~cm}^{\wedge} 2$

Length $=22.5=1.5 \mathrm{xd}$

Length is greater than 22.5 hence length $=30$

Compression Section: The Compression section proportion can fluctuate from 3-8 at low speed WT along these lines, as indicated by the length of the Compression section and compression proportion, as for the test area, the measurements of the section were chosen as:

Contraction proportion $=(40 \times 40) /(15 \times 15)=7$

Length $=40 \times 1.5 \mathrm{~d}$

Cross-section: 40x40 $\mathrm{cm}$ square (outside)

$15 \times 15 \mathrm{~cm}$ square (inside)

Length will accord to the comparative proportion of $\mathrm{L} / \mathrm{D}=1.5$

Honeycomb: The honeycomb takes after measurements of dimensions of Compression section from underlying point of view. In this manner,

Dimensions of Compression Section: $40 * 40 \mathrm{~cm}$ square

Thickness of the honeycomb ought to be greater than or equal to 2.2 in this will be. Approximately $5.9 \mathrm{~cm}$. in this way, with a specific end goal to limit turbulence we thought about the thickness as -Thickness: - 10cm (approx.)

Settling Section: As the honeycomb follows the measurements of the Compression section's underlying part, the similar manner settling section takes after the measurements of the honeycomb in this way, it has the indistinguishable area as that of the honeycomb.

Sectional Area: - 60*60 cm square (per screen)

Divergent Section: The Divergent section is composed by the diffuser angle of the Divergent section. It is composed with the end goal that the angle of diffusion $(\varphi)$ ought to be five degrees. In this way, we can also take $\varphi$ as: - 
$\varphi / 2=2.54$ Thus the external dia across (Do) of the Divergent section will be estimated

$\mathrm{D}_{0}=\mathrm{D}_{\mathrm{i}}+\{2 *(\mathrm{LD} * \tan \varphi / 2)\}$

Whereas, $D_{i}-$ inside cross section of Divergent section $=$ dia of test chamber $=15 \mathrm{~cm}$

$\mathrm{L}_{\mathrm{d}}-$ Divergent section length $=60$ centimeter so,

Do $=15+\{2 * 60 * \tan 2.54\}=15+7.984=21.984 \mathrm{~cm}$ (aprox.)

Fan: Since the WT we are developing, the Divergent section is at the outrageous section, accordingly, instead of an axial fan, we influenced utilization of the exhaust fan of half hp.

Targeted velocity in the test section $=200 \mathrm{~m} / \mathrm{sec}$

Mass flow rate in the test section $=\mathrm{PAV}=1.22 * 0.0225 * 200=5.51 \mathrm{~kg} / \mathrm{sec}$

Since, mass flow rate should be constant throughout all the section.

Hence, contraction section $=(\mathrm{p} \mathrm{X}$ a X v $)=5.51=1.225^{*} 0.16^{*} \mathrm{v}=5.51$

$\mathrm{V}=5.51 / 1.225 * 0.16=28.11 \mathrm{~m} / \mathrm{sec}$

Similarly, for diffuser:

$(\mathrm{pXa} X \mathrm{v})=5.51$

$\mathrm{V}=5.51 / \mathrm{aXp}=5.51 / 1.225 \times 0.0441$

$=101.99 \mathrm{~m} / \mathrm{sec}$

We should get above results in analysis

\section{MODELING AND ANALYSIS}

CATIA is software tool, which is used to do modeling of bodies.

CATIA offers a solution to shape design, styling, surfacing workflow and visualization to create, modify, and validate complex innovative shapes from industrial design to Class A with the ICEM surfacing technologies. CATIA supports multiple stages of product design, whether started from scratch or from 2D sketches.

\section{Procedure of Our Model}

Select $\mathrm{x} y$ plane and sketch, select the point axis, draw rectangular box, select work bench, select axis, then draw rectangular boxes simultaneously, totaling 5 rectangular boxes.

Next, go to work bench, select all rectangular boxes, next pad option join the rectangles

We get model solid.

Drafting: select home save file from mechanical, in that, select drafting, select views in, as per requirement with dimensions zoom in options to visible.

Then, save file CATIA part and IGS format to import into ANSYS tool

Software used ANSYS 18.2 
Steps and Procedure: Open ANSYS workbench, then select fluent in analysis system Geometry:

Import the geometry created in CATIA format, update it and then, geometry is imported to ANSYS.

\section{Meshing Steps}

Insert sizing > select contraction cone edges $>$ change the sizing to no. of divisions $>50>$ select

Behavior as hard >generate mesh. Now meshing for contraction cone is done.

Similarly, repeat the procedure for all the edges by inserting sizing at different intervals.

Mesh is generated then updates the project.

Now give named selection as follows:

- Contraction cone inlet as inlet

- Exit section of diffuser as outlet

- $\quad$ Select rest of surfaces as wall

Update project

Exit from the meshing.

\section{Solution}

Update the project in setup

Select energy equation and flow as viscous laminar.

Give boundary conditions according to the named selections.

Give inlet velocity as $28.11 \mathrm{~m} / \mathrm{sec}$ according to our calculations.

Initialize the solution in solution initialization

Give iterations as 100 and run the calculations

It takes a while to complete the calculations.

Select animations $>$ contours $>$ take required contours as follows

- Total pressure contour

- $\quad$ Static pressure contour

- Velocity magnitude contour

- Z velocity contour

Verify the values from Calculations.

Then analysis is done. 


\section{RESULTS}

Results from the analysis

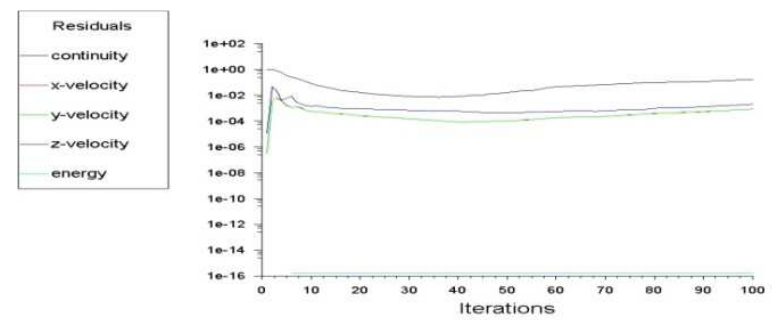

Figure (a): This Graph Explains the Results of Residuals Achieved for 100 Iterations from Analysis

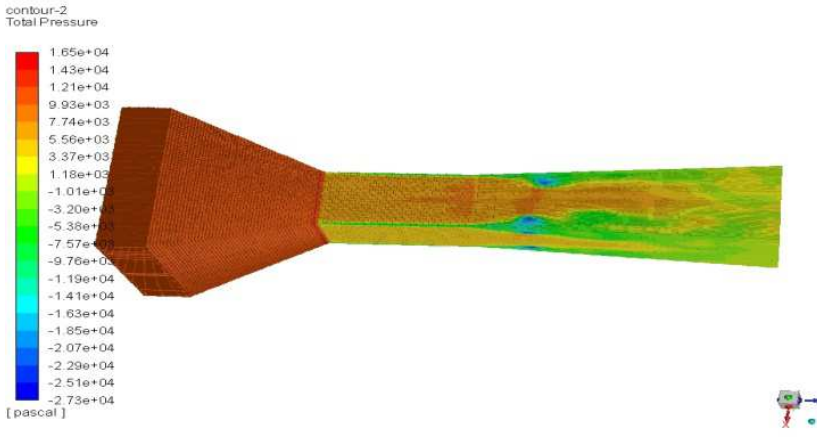

Figure (b): This Figure Shows the Total Pressure over the Wind Tunnel Passing from Convergent Section to Divergent Section
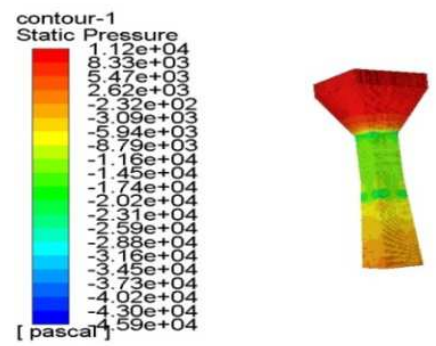

Figure (c): The above Figure Shows the Static Pressure over the Wind Tunnel, here we can Observe the Convergent Section is Highly Stressed but not Exceeding the Critical Values of the Wind

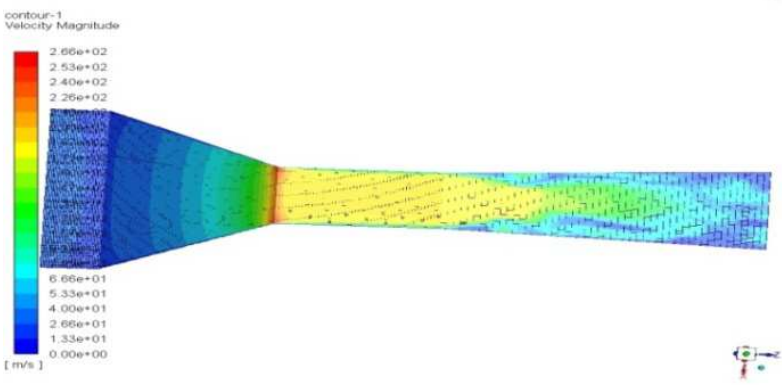

Figure (d): From Analysis we can observe the Velocity Magnitude Contour and we can Observe the End of the Convergent Section is highly stressed 


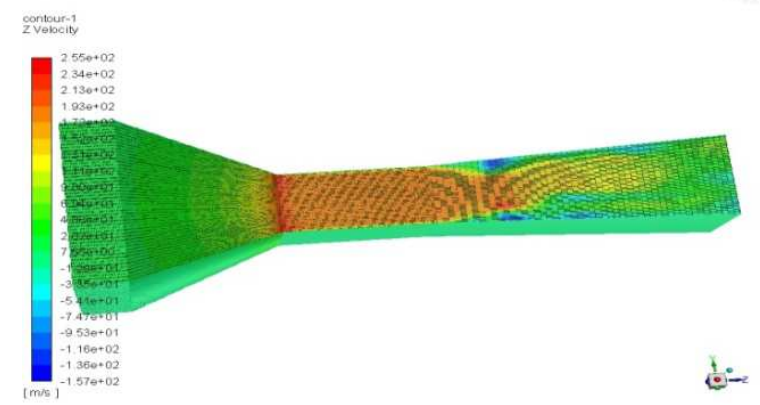

Figure (e): From Analysis the above Figure Explains the Flow Directions Passing from the Wind Tunnel Test Section and we can Observe the Most Affected Area by Color Coding

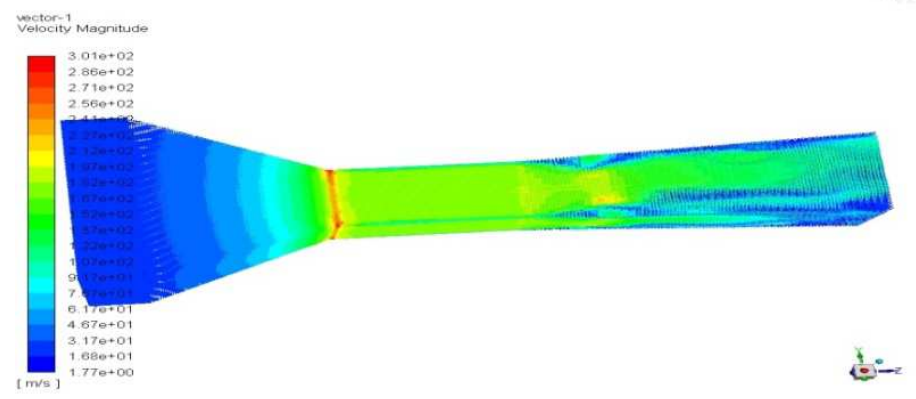

Figure (f): From Analysis the above Figure Explains the Velocity Magnitude of the Flow Passing from the Wind Tunnel Test Section

While verifying the results, we got the value of velocity, approximately equal to the theoretical calculations

\section{CONCLUSIONS}

- We have verified the problem statement

- Calculations for the model is done

- Catia model is prepared

- Analysis of the model is done

- Verification of the results is done

The WT facility gives a domain in which, significant learning takes place. Understudies need to learn, so they apply great engineering practice, for example, lab application of math and science, trial methodology and collaboration, to the utilization of WT. This kind of facility enables students to execute as engineers, and advances a rich learning experience, where, students take part in self-coordinated learning. The students would associate with the engineering process through utilization of the WT, and they will always thirst for more knowledge on the utilization and application of the engineering process.

\section{REFERENCES}

1. R. Mark Rennie, Peter Sutcliffe, Alexander Vorobiev, Alan B. Cain, "Mathematical modeling of wind speed transients in wind tunnels”, 51st AIAA Aerospace Sciences Meeting including the New Horizons Forum and Aerospace Exposition 07-10 January 2013, Grapevine (Dallas/Ft. Worth Region), Texas 
2. Tamura, T., "Numerical Study of Aerodynamic Behavior of a Square Cylinder," J. Wind Eng. Ind. Aerodyn, Vol. 33, pp. $161170(1990)$.

3. Md Khaleel, Marampalli Shilpa and L. Farooq, 2017. "Modeling and Cfd Analysis on One Stage of Turbine of Gas Turbine Engine”. International Journal of Civil Engineering \& Technology (IJCIET)

4. Shiva Prasad. U et.al., Design and Analysis of Two Throat Wind Tunnel, International Journal of Mechanical and Production Engineering Research and Development (IJMPERD), Volume 7, Issue 4, July - August 2017, pp. 381-388

5. STAR-CD version 3.15A manuals, Computational Dynamics Limited (2002).

6. Alka Sawale, Md Khaleel and S. Jaswanth, 2017. "Design and Analysis Of Winglet”. International Journal Of Civil Engineering \& Technology (IJCIET)

7. High Speed Wind Tunnel and Test Systems Design Handbook, Lockheed Martin Missiles and Fire Control, Texas, Publication Number Aer-Eir-13552-E.

8. K. Shiva Shankar, M. Satya Narayana Gupta and G. Parthasarathy, 2017. "Comparative Study of CFD Solvers for Turbulent Fuel Flow Analysis to Identify Flow Nature”. International Journal of Civil Engineering \& Technology (IJCIET) 
